\title{
Investigation of gold nanoparticle radiosensitisation mechanisms using a free radical scavenger and protons of different energies
}

\author{
J.C.G. Jeynes ${ }^{1{ }^{*}}$, M.J. Merchant ${ }^{1 *}$, A. Spindler ${ }^{1}$, A-C. Wera ${ }^{1}$, K.J.Kirkby ${ }^{1}$ \\ Ion Beam Centre, University of Surrey, Guildford, Surrey, UK \\ ${ }^{¥}$ Corresponding author: j.c.jeynes@surrey.ac.uk
}

*These authors contributed equally to this work

Keywords: linear energy transfer, clonogenic assay, Geant4, Monte Carlo

Abbreviations: Sensitiser Enhancement Ratio (SER), Relative Biological Effect (RBE), Gold nanoparticles (GNPs), Reactive Oxygen Species (ROS), Spread Out Bragg Peak (SOBP) 


\section{Abstract}

Gold nanoparticles (GNPs) have been shown to sensitise cancer cells to X-ray radiation, particularly at $\mathrm{kV}$ energies where photoelectric interactions dominate and the high atomic number of gold makes a large difference to X-ray absorption. Protons have a high cross-section for gold at a large range of relevant clinical energies, and so potentially could be used with GNPs for increased therapeutic effect.

Here, we investigate the contribution of secondary electron emission to cancer cell radiosensitisation and investigate how this parameter is affected by proton energy and a free radical scavenger. We simulate the emission from a realistic cell phantom containing GNPs after traversal by protons and X-rays with different energies. We find that with a range of proton energies ( $1-250 \mathrm{MeV})$ there is a small increase in secondaries compared to a much larger increase with X-rays. Secondary electrons are known to produce toxic free radicals. Using a cancer cell line in vitro we find that a free radical scavenger has no protective effect on cells containing GNPs irradiated with $3 \mathrm{MeV}$ protons, while it does protect against cells irradiated with X-rays. 


\section{Introduction}

Using nanoparticles to sensitise tumours to X-rays is an attractive proposition clinically as many nanoparticles (especially gold) are non-toxic and localise to tumours due to their increased vascularity (Khlebtsov and Dykman, 2011). Much research has focused on gold nanoparticles, but other types have been used such as titanium particles doped with rare earths (Townley, 2013, Townley et al., 2012b, Townley et al., 2012a), platinum NPs_(Hossain and Su, 2012), gadolinium and hafnium oxide NPs (Maggiorella et al., 2012) and quantum dots (Juzenas et al., 2008a). The major constraint in radiotherapy treatments is the damage to normal tissue. By increasing the local dose to the tumour by using nanoparticles, it is thought that the overall treatment dose could be reduced, thus decreasing unwanted side-effects. There have been many reports of using nanoparticles as radiosensitisers over the past decade, (Butterworth et al., 2012, Juzenas et al., 2008a). Most of the reports have been with relatively low energy (kV) X-rays, which have limited use clinically due to shallow penetration depth in the patient. X-rays with kV energy have a higher cross section for high $\mathrm{Z}$ materials such as gold because of photoelectric interaction, while Compton scattering and pair-production dominates with higher ( $\mathrm{MeV}$ ) X-rays where the $\mathrm{Z}$ number is not an important factor. Although there have been reports of radiosensitisation using GNPs in cancer cells with MeV beams used clinically (McMahon et al., 2011b, Jain et al., 2011), the effect is less dramatic than those seen with kV X-rays. With this in mind, protons (and carbon ions) offer an intriguing candidate for radiosensitisation with GNPs, as the cross section of high Z materials to protons is large at clinically relevant energies.

The mechanism of radiosensitisation from nanoparticles is yet to be fully elucidated. The most probable cause is Auger electron production from the surface of the nanoparticles, which leads to the creation of reactive oxygen species (ROS) which can result in cell death. Short range Auger electrons, rather than longer range photoelectric ejected electrons, are proposed to be the most significant contributor of ROS within the cell as they are very densely ionising (Juzenas et al., 2008a, Juzenas et al., 2008b). (Misawa and Takahashi, 2011), found seven times the concentration of $\mathrm{O}_{2}{ }^{-}$in irradiated water samples containing GNPs compared to controls, which they attributed to Auger electrons from the surface of the nanoparticles. DMSO has been used to elucidate the indirect effect of free radicals on cell killing with both X-rays and high-LET particles (Hirayama et al., 2009). 
In this paper, we use the DMSO method to assess the contribution of free radical cell death caused by irradiated nanoparticles in cells.

Radiotherapy with charged particles has become more prevalent in clinical practice over the past 20 years, and the possibility of radiosensitisation using GNPs is an attractive prospect. There have been a limited number of reports where relatively high energy particles (50 MeV or above) have been used to irradiate cells or tumours (Kim et al., 2012, Kim et al., 2010) containing nanoparticles showing radiosensitisation similar to those found with $\mathrm{X}$-rays.

In this paper, we irradiate cells containing GNPs with $3 \mathrm{MeV}$ protons and find no significant radiosensitisation. We compare these results with cells irradiated with X-rays where we show a significant radiosensitisation, which increases with gold content in the cell. Furthermore, we investigate the mechanism of this radiosensitisation. By using a free radical scavenger, we show that the radiosensitisation effect seen with $\mathrm{X}$-rays can be protected against, whereas this scavenger has no effects on the cell survival of cells irradiated with protons. In addition, we show using Geant4 Monte Carlo modelling that secondary electron emission from GNPs following interaction with protons of a range of energies (1-250 MeV), is insignificant compared to the interaction of GNPs and kV X-rays. We discuss our results with respect to other work where a radiosensitisation effect has been seen using high energy protons.

\section{Methods}

\section{Cell lines and culture}

For cell culture, RT112 bladder cancer cells (a gift from John Peacock, Kingston University, U.K.), were grown in RPMI 1640 media (Lonza, Wokingham, UK) with 10\% fetal bovine serum (FBS), $2 \mathrm{mM}$ glutamine and $100 \mathrm{IU} / \mathrm{ml}$ penicillin \& $1 \mathrm{mg} / \mathrm{ml}$ streptomycin incubated at 37 with $5 \% \mathrm{CO}_{2}$.

\section{Gold Nanoparticle treatment}

The 50nm GNPs are citrate stabilised colloids in pure water (British Biocell International, UK). Before incubating with the cells, the GNPs were either mixed with foetal bovine serum for nonspecific protein binding to the surface of the GNP, or conjugated to a TAT peptide. The details of this preparation are described elsewhere (Jeynes et al., 2013a), where we also show that the TAT 
coating compared to a FBS coating, increases the number of GNPs in the cell by about 5 times. Of note, it has been shown that coating the GNPs with bovine serum increases the diameter of the GNP by about 10nm (Tsai et al., 2011), while coating with TAT increases it by about 30nm (Patel et al., 2008).

The cells were seeded at $1 \times 10^{5}$ per $35 \mathrm{~cm}^{2}$ in tissue culture treated petri dishes, and incubated with $5.5 \mu \mathrm{g} \mathrm{ml}^{-1}$ GNPs-FBS or GNPs-TAT for 4 hours before irradiation. We chose this incubation time based on uptake studies by (Chithrani et al., 2006) who showed that the uptake rate plateaus after this time. The cells were then irradiated with either $250 \mathrm{kVp}$ X-rays or $3 \mathrm{MeV}$ protons.

\section{DMSO free radical scavenger treatment}

DMSO is an ideal free radical scavenger in living cells due to its low toxicity and high reactivity with the hydroxyl group $\left(\mathrm{OH}^{\cdot}\right)$. Irrad iated cells are co nf err ed a d egree of $p$ rot ect ion (D P) dep en $d$ in $g$ on the concentration of the DMSO. It has been shown that there is a plateau in the degree of protection above 1M DMSO (Shinohara et al.). Studies have shown that the indirect effect of X-ray radiation caused through the production of free radicals by water hydrolysis is about $75 \%$ of cell death. The other $25 \%$ is direct damage to the DNA which cannot be protected against. Charged particles are much more densely ionising than X-rays, and the contribution of direct action to cell killing is much greater. For example, with $16 \mathrm{MeV}$ iron particles about $70 \%$ of the cell death is caused by direct action (Hirayama et al., 2009)

Immediately prior to the irradiation with both X-rays and protons, the cells were incubated with media containing $1 \mathrm{M}$ of DMSO. This is non-toxic for short durations (Hirayama et al., 2009). Immediately after the irradiation, the cells are washed with PBS and incubated in normal fresh media (no DMSO).

\section{Confocal microscopy}

The cells were grown on glass coverslips overnight, and then incubated with GNPs for 4 hours. The cells were then fixed in $4 \%$ paraformaldehyde and the nuclei stained with propidium iodide. A Zeiss 510M confocal inverted microscope was used for imaging. A $514 \mathrm{~nm}$ laser was used to visualise the GNPs, using the surface plasmon resonance as described by Tsai et al., 2008. Optical stacks of $0.5 \mu \mathrm{m}$ were taken through the cells and analysed using Zeiss Zen software. 


\section{Proton irradiation}

Cells were irradiated using a $2 \mathrm{MV}$ Tandetron $^{\mathrm{TM}}$ High Voltage accelerator at the University of Surrey's Ion Beam Centre. The vertical beamline (Merchant et al., 2012) was used, with a procedure which has been described previously (Jeynes et al., 2013b). Here, the cells are pipetted onto a polypropylene-bottomed dish in a $15 \mu \mathrm{l}$ droplet and left for ten minutes for the cells to

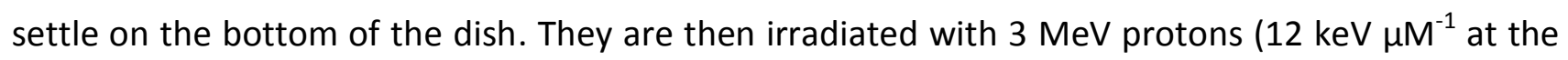
cell centre). To achieve the correct dose, the fluence of the beam is first checked with a PIN diode. The beam is then held over the droplet of cells for a precise length of time so that the desired dose is achieved. A $42 \mathrm{~mm}$ diameter petri dish contains up to 7 droplets each of which can contain cells treated with a different variable. After irradiation, the droplet containing cells is pipetted off the polypropylene, and diluted to the appropriate concentration for a clonogenic assay. We have found that this protocol did not affect the plating efficiency compared to cells cultured directly in tissue culture flasks.

\section{$X$-ray irradiation}

X-ray irradiation was performed using a Gulmay machine operating at $250 \mathrm{kVp}$ (Royal Surrey County Hospital, Guildford, UK), which is a polyenergetic beam. The cells were treated in exactly the same way as those irradiated with protons, except that 6 well plates were used rather than polypropylene-bottomed petri dishes. The plating efficiency of the cells irradiated with protons and that with $\mathrm{X}$-rays was the same $(\sim 40 \%)$ indicating that the method of irradiation did not affect the results.

\section{Clonogenic assay}

After irradiation, cells were seeded at an appropriate concentration for the dose received and grown in 6-well plates and incubated for up to 14 days. Colonies were fixed with $50 \%$ ethanol in PBS and then stained with 5\% crystal violet in PBS (Sigma-Aldrich, Dorset, UK). Colonies with more than 50 cells were counted and the survival fractions determined by taking into consideration the plating efficiency for all treatment modalities based on three separate experiments. 


\section{Geant4 Simulation}

The simulation was built using GEANT4.10.p01, CLHEP 2.1.3.1 and ROOT 5.28.00. The G4EmLivermorePhysics list was used for ion interactions with matter. The secondary electrons and secondary X-rays that were produced from each incident particle or photon, were tracked with a cut-off of $10 \mathrm{eV}$. All electrons were tracked including those that arose from cascade events. The energy of all the secondary electrons produced from incident particles or photons was compared between a water phantom geometry containing GNPs and one without. The geometry was modelled as a $64 \mu \mathrm{m}^{3}$ cube of water. Within it, spheres representing vesicles were randomly distributed, and each vesicle was filled with a number of GNPs (see Figure 3 and Results). We have estimated uncertainty in these simulations by running the monte carlo simulation 100 times with identical parameters and measuring the total number of secondary electrons emitted. For example, the total number of electrons over the 100 separate runs for $250 \mathrm{MeV}$ protons has a mean of 13595 electrons and a standard deviation of 582 electrons, giving a $4.28 \%$ uncertainty. With $50 \mathrm{MeV}$, there are 51907 and a standard deviation of 1334 electrons giving an uncertainty of $2.6 \%$.

\section{Statistical analysis}

All of the experiments were repeated in triplicate on at least two separate days. The results are averaged and the standard deviation is given. All curve fittings were performed in OriginPro (Northampton, US) with an optimisation of the fit weighted to the variance of the data points.

Where appropriate, statistical significance was determined using analysis of variance (ANOVA) and a Tukey test to compare variables. $P$-values less than 0.05 were considered significant.

The Linear-Quadratic equation was used to fit clonogenic survival data:

Where $\alpha$ defines the linear component, $\theta$ relates to the quadratic component, and $x$ is the dose.

The Sensitiser Enhancement Ratio (SER) was given using the following formula: 
Where dose $_{x \%}$ (no GNPs) is the radiation dose (Gy) required to produce $x \%$ cell survival without GNPs and dose $_{x \%}$ (GNPs) in the presence of GNPs. SER was calculated at doses related to surviving fractions of $50 \%$.

\section{Results}

GNP localisation within cells

Figure 1 shows two optical stacks from the bottom of the cell (where it is attached to the glass slide) and one near the top of the cell, just above the nucleus. These cells were incubated with $50 \mathrm{~nm}+\mathrm{TAT}$, and contain on average 5,000 GNPs per cell (please see gold quantification in (Jeynes et al., 2013a). The GNPs can be seen as red dots in the image, which contain many individual GNPs in vesicles. The nucleus of each cell can be seen in green in the centre of each cell. Interestingly, in the stack through the nucleus (Figure 1a) GNPs are seen throughout the cytoplasm of the cell, with an insignificant number within the nucleus itself. It is clear from the stack just above the nucleus (Figure 2b) that nanoparticles are in the cytoplasm above the cell nucleus.

\section{Radiosensitisation using GNPs with X-rays and protons}

Figure 2A shows a survival curve up to $8 \mathrm{~Gy}$ comparing control cells to those incubated with $50 \mathrm{~nm}+\mathrm{FBS}$ GNPs. The data is fitted with the Linear Quadratic model giving $\alpha$ and $\beta$ parameters which are shown in table 1 . The $\alpha$ parameter with the cells incubated with GNPs is larger, and consequently there is less of a shoulder on the curve. The $\beta$ value is slightly smaller in the GNP incubated cells, indicating a shallower slope. The sensitiser enhancement ratio at $50 \_$survival is $1.6 \pm 0.2$.

Figure 2B shows cells irradiated with $5 \mathrm{~Gy}$ of $250 \mathrm{kVp}$ X-rays, with various conditions. In previous work, we showed that conjugating a TAT peptide to GNPs increased the numbers of GNPs in the cells by about 5 times, from an average of about 1000 GNPs per cell without the peptide to about 5,000 GNPs with the TAT peptide (Jeynes et al., 2013a). Figure 2b shows that incubating with $50 n m+F B S$ GNPs decreases cell survival by about a $50 \%$ compared to the control. By incubating with the 50nm+TAT GNPs this decreases by about 300\% compared to the control. By incubating cells with DMSO, a free radical scavenger, cell survival increases by about 60-250\% compared to the untreated DMSO counterpart. The statistical significance of these results was tested using ANOVA (see Figure 2 legend). 
Figure $2 \mathrm{C}$ shows cells irradiated with $5 \mathrm{~Gy}$ of $3 \mathrm{MeV}$ protons with the same conditions as shown in Figure 2B. Contrastingly to cells irradiated with $\mathrm{X}$-rays, there is no significant difference between the control and the different conditions, using an ANOVA test.

\section{Modelling secondary electron production with Geant4}

Figure 3 shows the geometry used in the Geant4 simulation of the secondary electron production from incoming $X$-rays or ions. The design of this geometry is based on data from-(Peckys and de Jonge, 2011t, which measured GNPs in clusters held within vesicles in the cytoplasm of cells using an environmental scanning electron microscope. In our simulation, a cube with $4 \mu \mathrm{m}$ sides (64 $\mu \mathrm{m}^{3}$ ) was constructed containing spheres or "vesicles" which were filled with GNPs. There are 135 vesicles in total which vary in diameter from $150 \mathrm{~nm}$ to $350 \mathrm{~nm}$, and are randomly distributed through the cube. A total of 1000 GNPs are distributed randomly throughout these vesicles.

Figure 4 shows the difference in secondary electrons compared to a control, after the phantom containing GNPs are irradiated with $10^{6}$ particles with different initial energies of protons or X-rays with a -distribution of energies from an orthovoltage $250 \mathrm{kVp}$ linak. For both the X-rays and the proton simulations, over 10,000 secondary electrons were generated in total for each energy (with the lower energy protons creating considerably more). All electrons are tracked whether they are created by the incident beam or by cascade events. A range cut was established to prevent the counting of any electrons which had a predicted range below the size of the nanoparticle diameter, so that electrons trapped inside the nanoparticles were not added to the final totals. The electrons were tracked to an energy of $10 \mathrm{eV}$ which is the limit of the version of Geant4 that we were implementing.

Figure 4A shows a histogram of the energy of secondary electrons emitted from $10^{6}$ primary X-rays measured in the simulation, with and without GNPs. It is clear that without the GNPs there are very few secondary electrons emitted, whereas with the GNPs secondary electrons are produced mainly at $15 \mathrm{keV}$, but some having energies as high as $200 \mathrm{keV}$. Figure 4B and C shows a similar histogram but with 2000, $3 \mathrm{MeV}$ and $250 \mathrm{MeV}$ protons as the incident particle. Here there is a much smaller difference compared to the X-rays between the 
numbers of the secondary electrons with or without the GNPs. The results shown in Figure 4D show that X-rays give about a 3 fold increase in the emitted secondary electrons, while protons give between 1.01-1.15 fold increase depending on the initial energy.

\section{Discussion}

In this study, we have shown that that GNPs contained in the vesicles of cancer cells enhance X-ray cell killing, while there is no significant effect for low energy protons. The novelty of this work lies in the experimental use of protons and X-rays with different preparations of GNPs and free radical scavengers, and the simulation of the experiment using a realistic Geant4 simulation. Simulating the secondary electron emission in realistic cell geometry with and without GNPs, showed that Xrays produced about a 3 fold secondary electron emission increase, whereas with protons the increase was marginal.

There have been many reports of sensitisation of cells in vitro and in vivo using gold nanoparticles with X-rays (Butterworth et al., 2012). Between the experimental studies, various parameters differ such as; the cell line, the GNP surface coating, the GNP concentration, the incubation time and the GNP diameter. However, the clear trend is that sensitisation is seen to varying degrees with different cell types and GNPs preparations. Interestingly, the predicted dose enhancement based on total extra dose deposited due to gold being present, is orders of magnitude less than is actually observed (Lechtman et al., 2011). This is true even of MV X-rays where Compton interactions or pair production dominate and very little extra dose is expected.

The reason for the disparity between macroscale simulation and experimental data has been investigated by simulating the density of the secondary electrons produced from GNPs on a nanometer scale using Geant4. MEMahon et al., 2011a and Leung et al., 2011, simulated secondary electron emission from individual GNPs and postulated that local dose enhancement from short range Auger electrons mimicked the enhanced relative biological effect of heavy ions. With heavy ions, a comparable dose can be deposited to an equivalent dose of X-rays but the survival fraction of cells is much lower (Hirayama et al., 2009). The dose is localised to the ion- track and results in many more lethal double-strand breaks than the same dose spread 
homogenously. However, radiosensitisation based on this hypothesis would rely on the GNPS being physically close to the DNA, so that short range Auger electrons could have any direct effect. Very few reports have simulated a virtual cell with GNP localised to different parts of the cell with Geant4,_with some notable exceptions. (Douglass et al., 2013) measured the sensitiser enhancement ratio (SER), when GNPs where placed at various organelles. They found that the SER in the nucleus was as high as 55 if the GNPs were placed in the nucleus whereas it was only about 7 in the cytoplasm. fLechtman et al., 2013 t modelled a nanoscale rather than a macroscopic deposition, and were able to match the simulated sensitiser enhancement ratio of about 1.2 with their experimentally obtained value. Wazlein et al., 2014 simulated up to a 2 fold dose enhancement for heavy atom nanoparticles irradiated with 2, 80 and $300 \mathrm{MeV}$ protons. This was attributed to Auger electrons, as the enhanced dose only existed within nanometers from the surface of the NPs.

Interestingly, none of the previously mentioned simulations takes into account the production of reactive oxygen species, which undoubtedly have a major contribution to cell death. This is a limitation of the current Geant4, but which is being updated in a new code "Geant4DNA", which implements water radiolysis algorithms (Sepan and Davidkova, 2008). By using DMSO as a free radical scavenger, we directly show that the death associated with X-rays is, at least in part, caused by reactive oxygen species, and that this effect is accentuated when GNPs are present. Moreover, we show that the ROS species have little effect on the cell death mechanism when cells are irradiated with $3 \mathrm{MeV}$ protons, and that the GNPs have no additional effect. It is well know that high-LET radiation incurs most damage through direct action rather than indirect action. (Bishayee et al., 2000) showed that DMSO protected cells from ${ }^{125} \mathrm{I}$-and ${ }^{131}$ I-iododeoxyuridine $\left({ }^{125} \mathrm{IdU},{ }^{131} \mathrm{IdU}\right) \quad \beta$-emitters, but not against ${ }^{210} \mathrm{Po}$ emitting $5.3 \mathrm{MeV} \alpha$ particles. Various experimenters have shown that DMSO can protect about $75 \%$ of the damage from $\mathrm{X}$-rays (Hirayama et al., 2009, Shinohara et al., 1996). Interestingly, the degree of protection that DMSO affords high-LET radiation, depends on the energy (or LET) of the particle, with those near the Bragg peak imparting much more direct-action damage as one would expect. (Hirayama et al., 2009+ showed that DMSO can protect about $50 \%$ of the cell death caused by $500 \mathrm{MeV}$ Iron ions, but only about 30\% with $16 \mathrm{MeV}$ particles. As we show that the GNPs are not significantly within the nucleus (Figure 1), we suggest that generated ROS either traverse across the nuclear membrane and attack DNA directly, or degrade other crucial proteins within the cytoplasm. (Sicard-Roselli et al., 2014). 
Intracellular damage by reactive oxygen species is a topic that has received much attention over the past few decades (Silva and Coutinho, 2010). The ROS that are generated do not have to react directly with DNA to cause cell death. Excessive ROS can react with a variety of cell biomolecules in the cytoplasm (lipids, proteins, enzymes) which ultimately disrupt organelle function and can cause either apoptosis or necrosis by one of the various pathways that exist in these cell death mechanisms. That said, due to the sensitivity of nucleus we expect that GNPs located there will result in additional cell death.

We irradiated cells with a polyenergetic X-ray beam of with a maximum energy of $250 \mathrm{kV}$ and an energy distribution based on information from the manufacturer (Gulmay). There are studies which investigate the effect of the energy of X-rays on radiosensitisation with GNPs both experimentally and using simulation. Leung et al. 2011, simulated polyenergetic X-rays and found that the interaction enhancement ratio went in this order with highest first: $50 \mathrm{kVp}, 250 \mathrm{kVp}, 6$ $M V p$, cobalt 60 source. The reason for this is a strong energy dependence of the photoelectric interaction cross section for gold at $79 \mathrm{kV}$. Experimentally, it has been shown that more radiosensitisation is seen with $250 \mathrm{kVp}$ X-rays compared to $6 \mathrm{MVp}$ X-rays (Jain et al. 2011).

There have been limited reports of radiosensitisation with ions combined with nanoparticles. Moreover, all investigations undertaken so far have been with high energy ions above $50 \mathrm{MeV}$ with none that we know of with low-energy ions. Low-energy ions are of interest clinically because a proportion of beam energy in the spread out Bragg peak will be below $10 \mathrm{MeV}$. Only two reports have been performed in vivo._Polf et al., 2011 used a $160 \mathrm{MeV}$ proton beam irradiating cells with internalised GNPs, and found an enhancement of about $20 \%$ compared to proton irradiated controls. Kaur et al., 2013-used $62 \mathrm{MeV}{ }^{12} \mathrm{C}^{6+}$ and found that using GNPs increased cell killing by about $40 \%$. One group has investigated the effects of GNPs in vivo with charged particles. Kim et al., 2010-irradiated tumours injected with gold or iron nanoparticles with a $41.7 \mathrm{MeV}$ proton beam, and found significant reduction in the tumour volume compared to the control. This work was followed by another paper which found the reactive oxygen species in irradiated tumour cells containing nanoparticles was 12-36 \% higher than in the control (Kim et al., 2012)--

It is interesting that other researchers have seen an enhancement using protons and nanoparticles, while we did not. This is most likely due to the energy of the ions interacting with nanoparticles. With proton beam energies above $40 \mathrm{MeV}$, the LET of the ions as it traverses the 


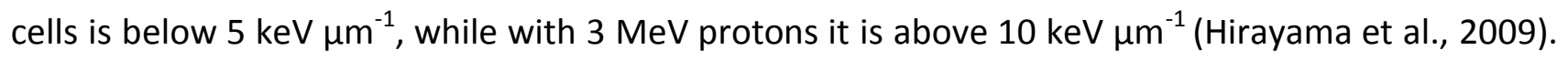
The LET of the ion determines the dose it imparts, consequently cells are irradiated with a far greater number of low-LET ions for the same equivalent dose of high-LET particles (Jeynes et al., 2013b). Therefore the probability of an ion hitting a vesicle containing nanoparticles is greater with higher energy, low-LET protons, as there are more ions traversing the cell. Moreover, the energy of the primary ion will determine the energy of the emitted secondary electron. With high energy ions, most of the secondary electrons will escape the nanoparticle and consequently produce reactive oxygen species which can disrupt cellular processes.

We have simulated the energy spread of the Bragg peak of a $250 \mathrm{MeV}$ beam in water using TRIM (Ziegler et al., 2010). The height of the Bragg peak has an average energy of $19.8 \pm 8.3 \mathrm{MeV}$. This means that most of the tumour tissue will be irradiated with relatively high energy beams (>10 $\mathrm{MeV})$. According to our simulations there will be a modest increase in the numbers of secondary electrons emitted if the tumour contains NPs, which potentially could increase cell killing. It is important to note that GNPs localise in tumours over normal tissue, due to the tumours increased vascularity and leaky capillary network. It has been shown in rodents that the blood system quickly clears GNPs from most organs and tissues, while GNPs are retained in tumours (and some other organs like the kidneys). In this way, normal tissue would have little radiosensitisation from the GNPs as there would be very low levels of GNPs present.

In this study we have used a single cell line. In future experiments we plan to extend the study with other tissue types. Some variation in the extent of the effects of radiosensitisation is expected in other cell lines related to the sensitivity of the particular cell type.

\section{Conclusions}

In this paper, we have combined experimental data with simulation and showed that radiosensitisation caused by GNPs and X-rays,-is most likely caused by secondary electrons resulting in extra reactive oxygen species. In contrast to this, protons of a range of energies only produce modest amounts of extra secondary electrons when interacting with GNPs, Through scavenging free radicals with DMSO, we have shown that at least some of the damage caused by X-ray irradiation of GNPs can be protected against, while it has no 
effect with $3 \mathrm{MeV}$ protons. Further studies are needed to confirm this, especially with higher energy protons, and to find possible other mechanisms by which GNPs can radiosensitise cells.

\section{Acknowledgements}

This work has been supported by the European Community as an Integrating Activity «Support of Public and Industrial Research Using Ion Beam Technology (SPIRIT)» under EC contract no. 227012, by SPRITE, and by the UK EPSRC under grant EP/C009592/1. We thank the Royal Surrey County Hospital (UK) for providing beamtime for the X-ray experiments.

\section{Conflict of interest}

The authors do not have any conflicts of interest to report. 


\section{FIGURES}
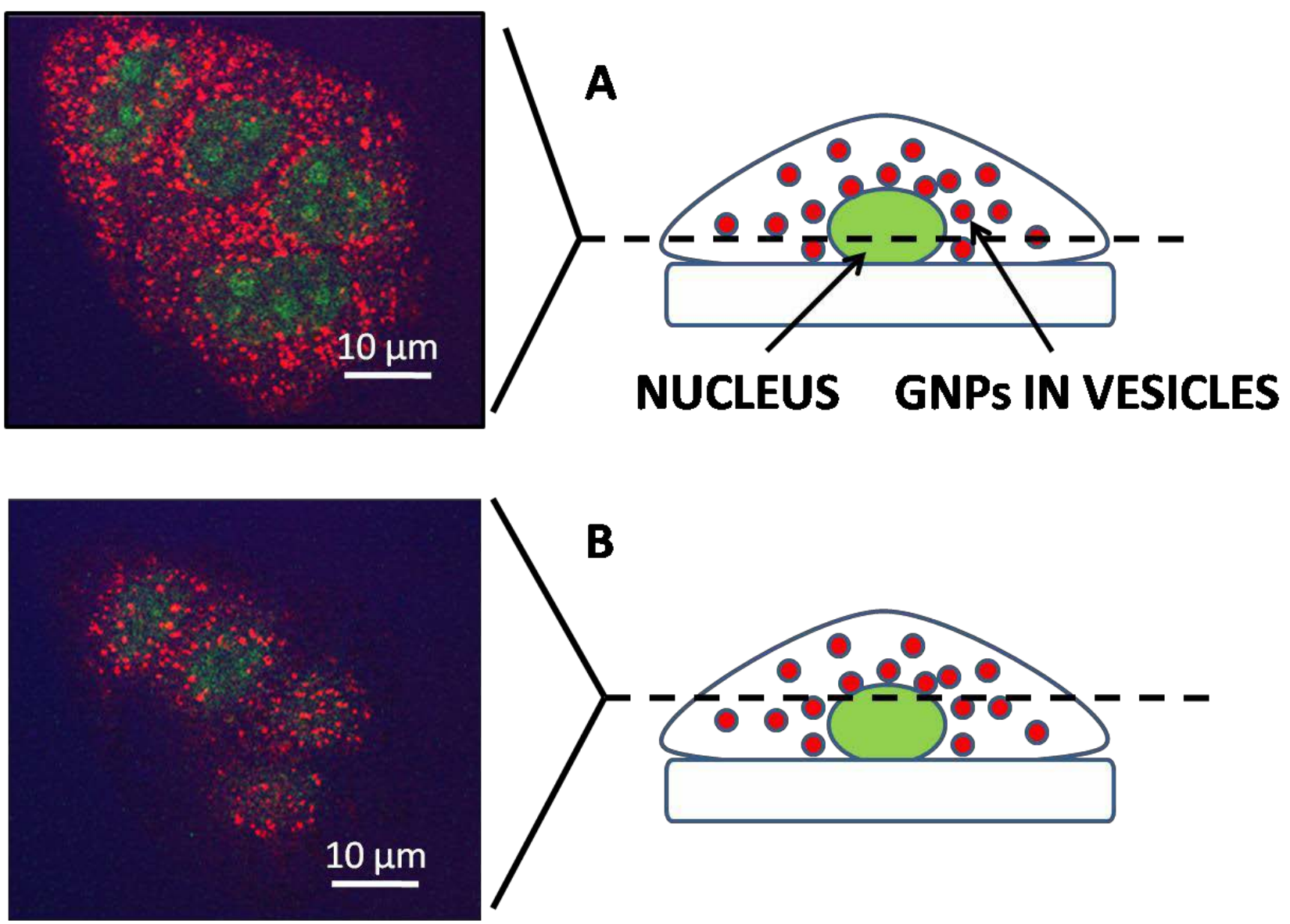

Figure 1: Confocal optical stack through cells to show the position of the gold nanoparticles within the cells. The cells have been incubated for 4 hours with 50nm+TAT GNPs. These images show a cluster of 4 cells tightly packed together. A shows an optical slice which is taken through the centre of the nucleus, indicated by the dashed line. B shows an optical slice which is taken at the very top of the nucleus. The images show that the gold nanoparticles (contained in vesicles) are predominantly located around the nucleus, but do not enter it. 


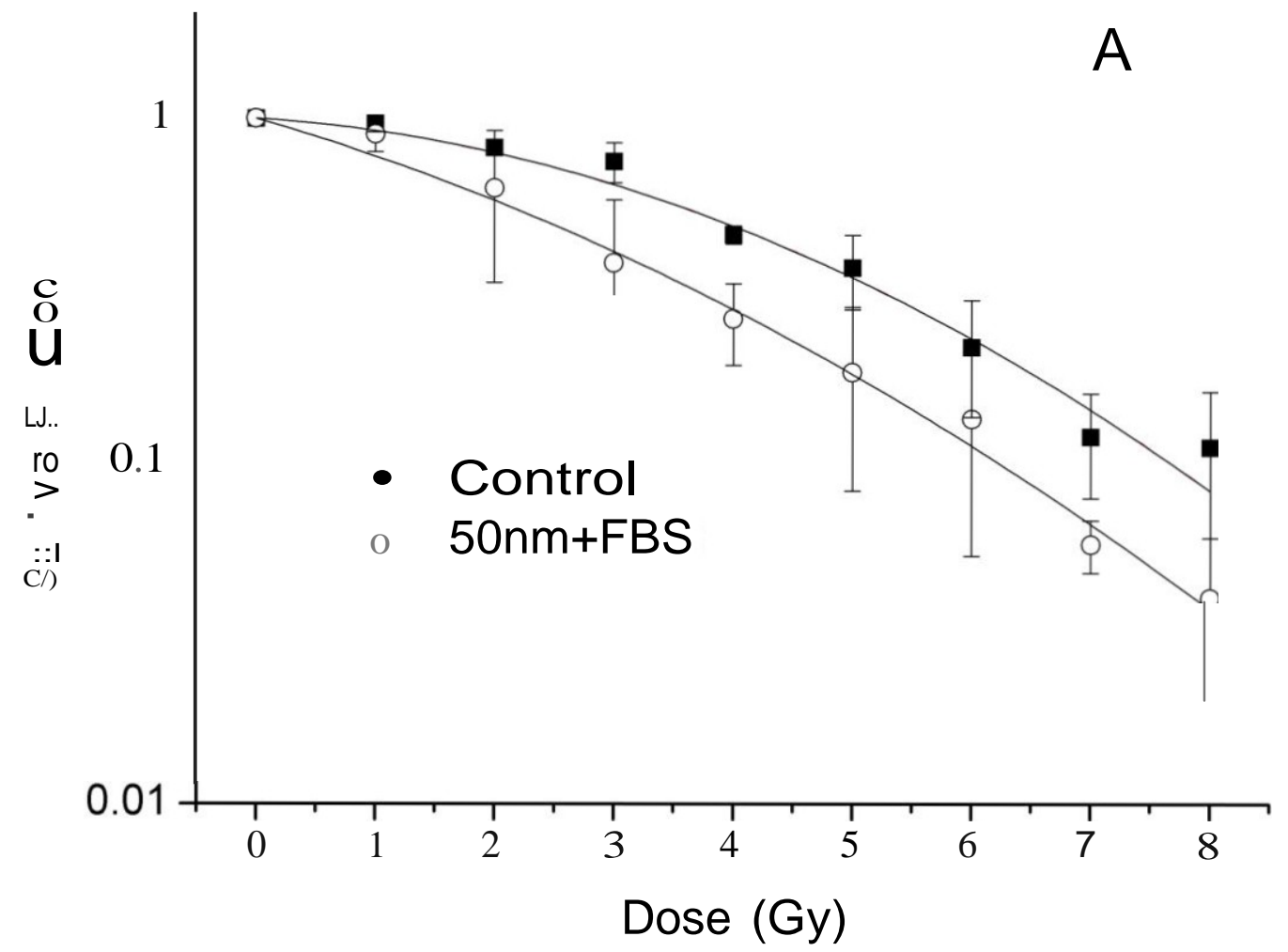

$0.7 \quad 5$ Gy, 250 kVp X-rays

8

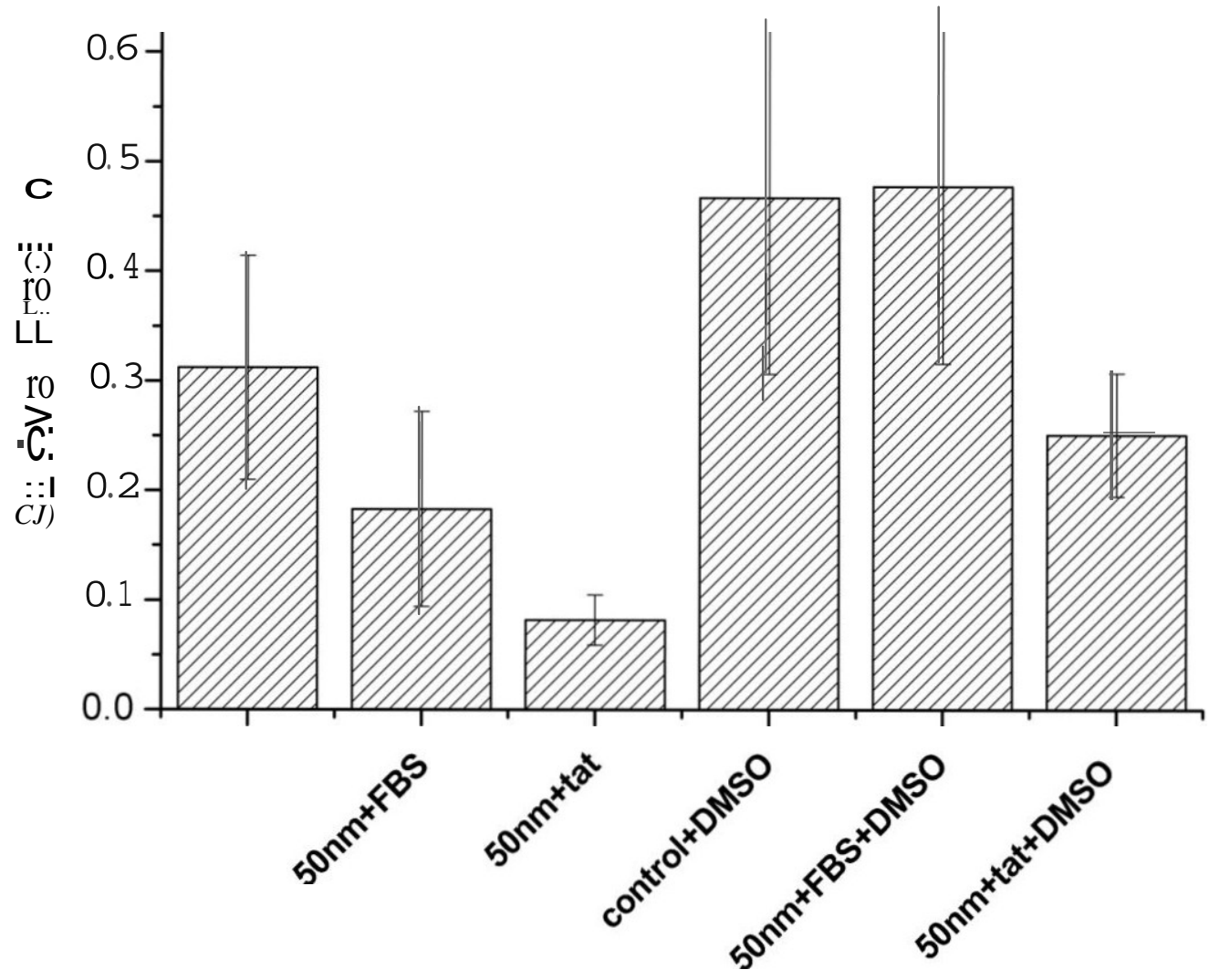




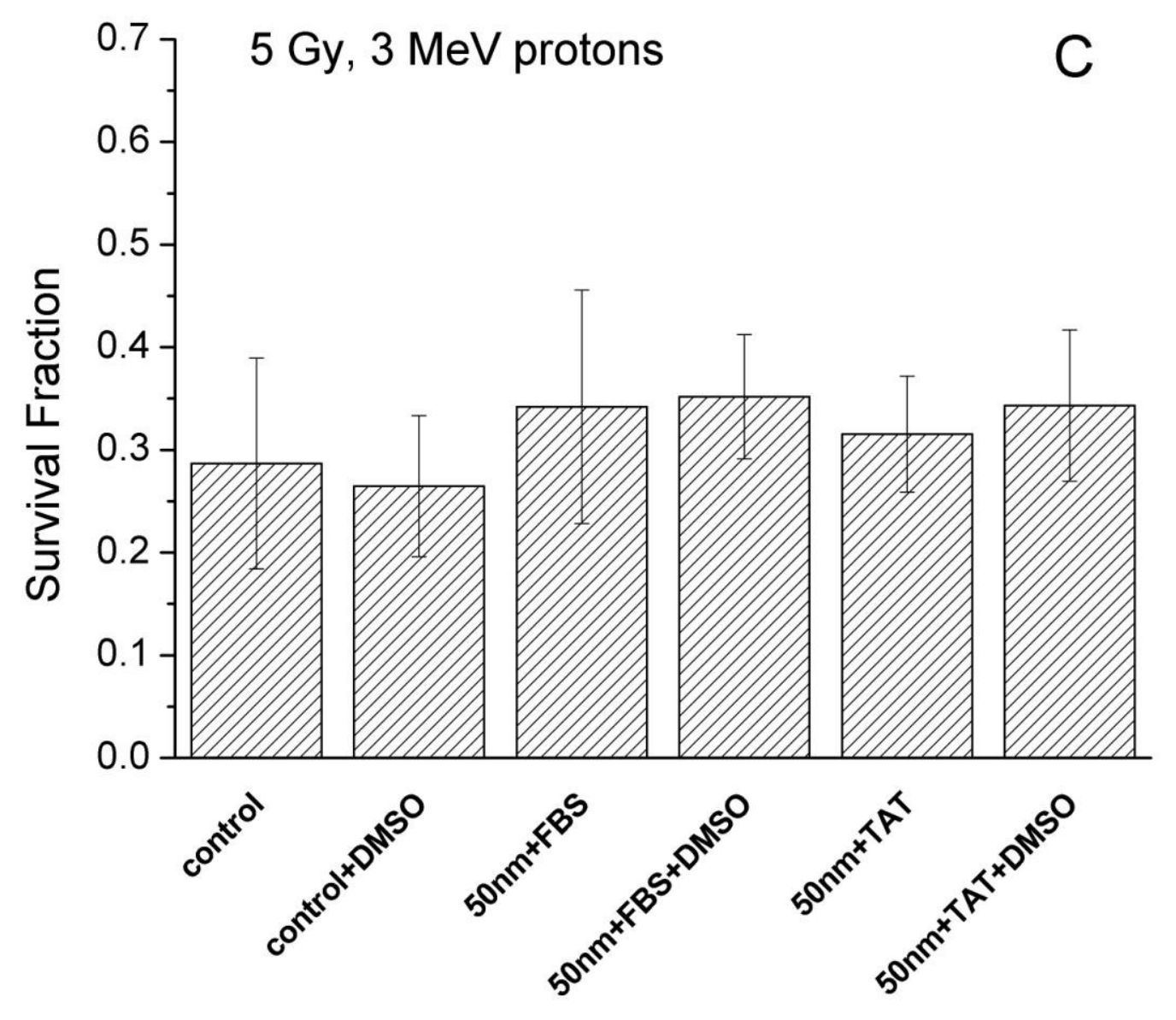

Figure 2: A Survival fraction of RT112 cells with $250 \mathrm{kVp}$ X-rays incubated with 50nm+FBS GNPs compared to a control. B Survival fraction at 5 Gy $250 \mathrm{kVp}$ X-rays comparing cells incubated with $50 \mathrm{~nm}+\mathrm{FBS}$ or $50 \mathrm{~nm}+\mathrm{TAT}$ GNPs, and DMSO which is a reactive oxygen species scavenger. There is on average 5 times the number of GNPs in cell incubated with 50nm+TAT (Jeynes et al. 2013). The control is significantly different from $50 \mathrm{~nm}+\mathrm{TAT}$ at $P=0.05$ using a one-way ANOVA, while the control and 50nm+FBS are different at $P=0.1$. The DMSO treated cells are all significantly different from their non-DMSO counterparts at $P=0.05$. C Survival fraction at $5 \mathrm{~Gy}, 3 \mathrm{MeV}$ protons. Using a one way ANOVA test, none of these conditions are significantly different at $P=0.05$ level. 


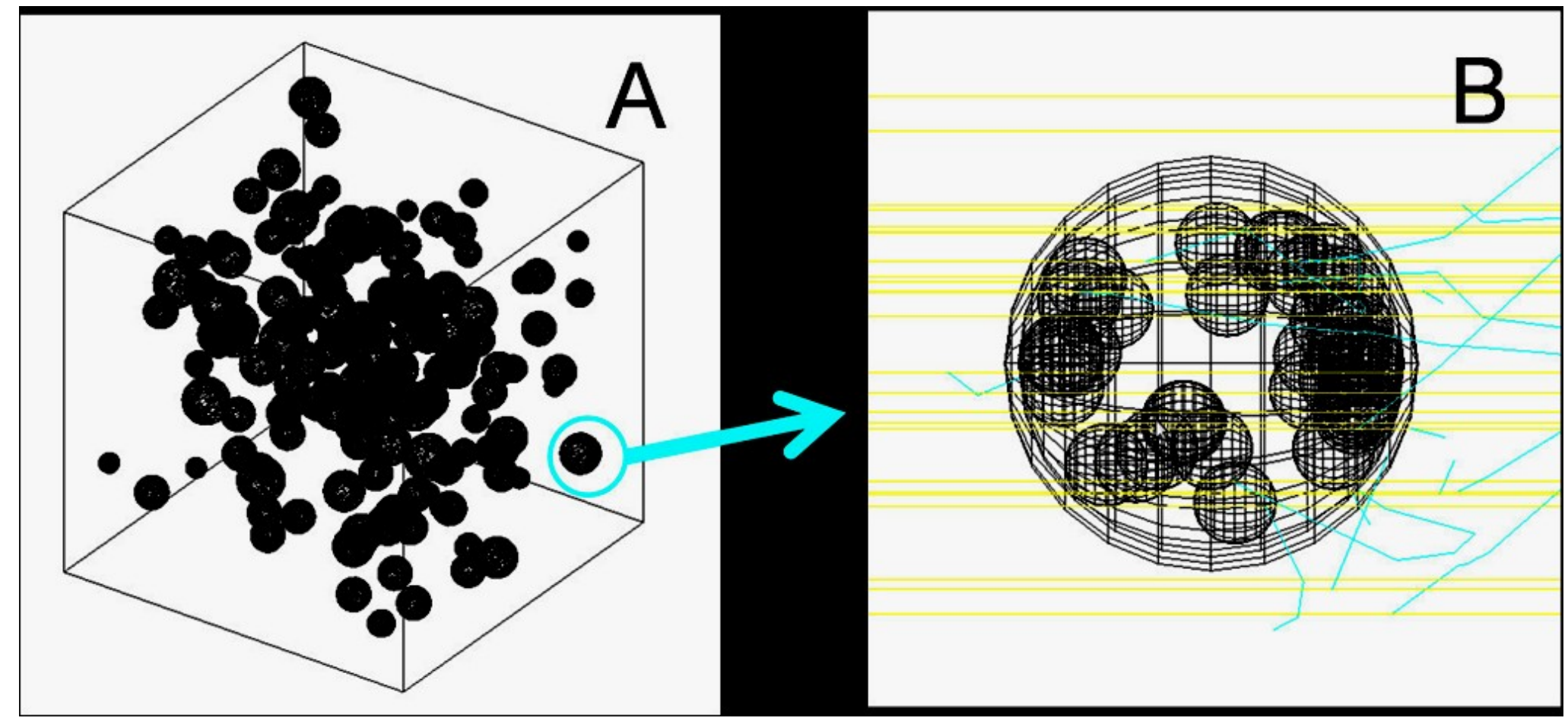

Figure 3: Geant4 modelling schematic. A shows the Geant4 geometry, which is a $64 \mu \mathrm{m}^{3}$ phantom filled with water in which secondary electron energies are tracked. Each sphere is a simulated "cell vesicle" containing gold nanoparticles. The "vesicles" are randomly distributed and the number of GNP contained within them is between 30-100 GNPs. B magnified image of an individual "vesicle" containing GNPs (smaller spheres). The straight yellow lines are protons which pass through the "vesicles" inducing secondary electron emission (blue lines) from the GNPs. 

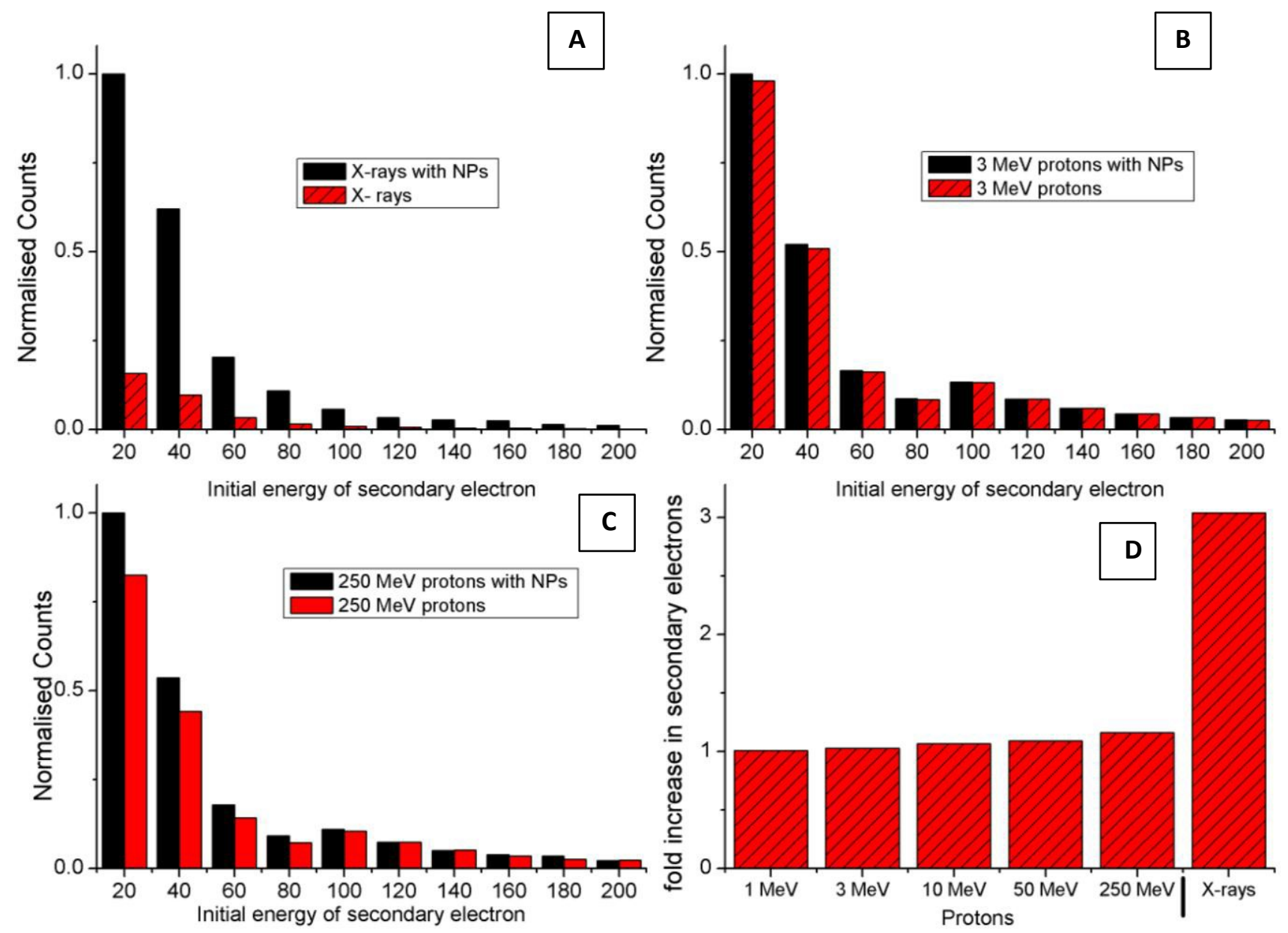

Figure 4: Compares the initial energy of secondary electrons produced from a primary beam of either (A) $10^{6}$, X-rays (realistic distribution of energies from an orthovoltage $250 \mathrm{kVp}$ linak) or (B) 2000, $3 \mathrm{MeV}$ protons produced in the geometry (see figure 3) either with or without gold nanoparticles. (C) $250 \mathrm{MeV}$ protons. All results have been normalised for easy comparison, but each bin contains at least 100 electron counts (D) The total increase in secondary electrons emitted from the phantom containing NP compared to the control for a range of proton energies and X-rays. In all of the simulations, a total of at least $10^{4}$ electrons were produced. 
Table 1: Radiobiological parameter values

\begin{tabular}{|ccc|}
\hline \multicolumn{3}{|c|}{ Control } \\
\hline$\alpha$ & 0.048 & \pm 0.042 \\
$\beta$ & 0.033 & \pm 0.007 \\
\hline \multicolumn{3}{|c|}{$50 \mathrm{~nm}$ GNPs } \\
\hline$\alpha$ & 0.229 & \pm 0.032 \\
$\beta$ & 0.023 & \pm 0.005 \\
\hline
\end{tabular}




\section{References}

BISHAYEE, A., RAO, D. V., BOUCHET, L. G., BOLCH, W. E. \& HOWELL, R. W. (2000) Protection by DMSO against cell death caused by intracellularly localized iodine-125, iodine-131 and polonium-210. Radiation Research, 153, 416-427.

BUTTERWORTH, K. T., MCMAHON, S. J., CURRELL, F. J. \& PRISE, K. M. (2012) Physical basis and biological mechanisms of gold nanoparticle radiosensitization. Nanoscale, 4, 4830-4838.

CHITHRANI, B. D., GHAZANI, A. A. \& CHAN, W. C. W. (2006) Determining the size and shape dependence of gold nanoparticle uptake into mammalian cells. Nano Lett. , 6, 662-668.

DOUGLASS, M., BEZAK, E. \& PENFOLD, S. (2013) Monte Carlo investigation of the increased radiation deposition due to gold nanoparticles using kilovoltage and megavoltage photons in a 3D randomized cell model. Medical Physics, 40.

HIRAYAMA, R., ITO, A., TOMITA, M., TSUKADA, T., YATAGAI, F., NOGUCHI, M., MATSUMOTO, Y., KASE, Y., ANDO, K., OKAYASU, R. \& FURUSAWA, Y. (2009) Contributions of Direct and Indirect Actions in Cell Killing by High-LET Radiations. Radiation Research, 171, 212-218.

HOSSAIN, M. \& SU, M. (2012) Nanoparticle location and material dependent dose enhancement in X-ray radiation therapy J. Phys. Chem. C, 116, 23047-23052.

JAIN, S., COULTER, J. A., HOUNSELL, A. R., BUTTERWORTH, K. T., MCMAHON, S. J., HYLAND, W. B., MUIR, M. F., DICKSON, G. R., PRISE, K. M., CURRELL, F. J., O'SULLIVAN, J. M. \& HIRST, D. G. (2011) Cell-Specific Radiosensitization by Gold Nanoparticles at Megavoltage Radiation Energies. International Journal of Radiation Oncology Biology Physics, 79, 531-539.

JEYNES, J. C. G., JEYNES, C., MERCHANT, M. J. \& KIRKBY, K. J. (2013a) Measuring and Modelling cell-to-cell variation in the uptake of gold nanoparticles. Analyst, 138, 7070-4.

JEYNES, J. C. G., MERCHANT, M. J., BARAZZUOL, L., BARRY, M., GUEST, D., PALITSIN, V. V., GRIME, G. W., TULLIS, I. D. C., BARBER, P. R., VOJNOVIC, B. \& KIRKBY, K. J. (2013b) "Broadbeam" irradiation of mammalian cells using a vertical microbeam facility. Radiation and Environmental Biophysics, 52, 513-521.

JUZENAS, P., CHEN, W., SUN, Y.-P., NETO COELHO, M. A., GENERALOV, R., GENERALOVA, N. \& CHRISTENSEN, I. L. (2008a) Quantum dots and nanoparticles for photodynamic and radiation therapies of cancer. Advanced Drug Delivery Reviews, 60, 1600-1614.

JUZENAS, P., GENERALOV, R., JUZENIENE, A. \& MOAN, J. (2008b) Generation of Nitrogen Oxide and Oxygen Radicals by Quantum Dots. Journal of Biomedical Nanotechnology, 4, 450-456.

KAUR, H., PUJARI, G., SEMWAL, M. K., SARMA, A. \& AVASTHI, D. K. (2013) In vitro studies on radiosensitization effect of glucose capped gold nanoparticles in photon and ion irradiation of HeLa cells. Nuclear Instruments \& Methods in Physics Research Section B-Beam Interactions with Materials and Atoms, 301, 7-11.

KHLEBTSOV, N. \& DYKMAN, L. (2011) Biodistribution and toxicity of engineered gold nanoparticles: a review of in vitro and in vivo studies. Chemical Society Reviews, 40, 1647-1671.

KIM, J.-K., SEO, S.-J., KIM, H.-T., KIM, K.-H., CHUNG, M.-H., KIM, K.-R. \& YE, S.-J. (2012) Enhanced proton treatment in mouse tumors through proton irradiated nanoradiator effects on metallic nanoparticles. Physics in Medicine and Biology, 57.

KIM, J.-K., SEO, S.-J., KIM, K.-H., KIM, T.-J., CHUNG, M.-H., KIM, K.-R. \& YANG, T.-K. (2010) Therapeutic application of metallic nanoparticles combined with particle-induced $\mathrm{x}$-ray emission effect. Nanotechnology, 21.

LECHTMAN, E., CHATTOPADHYAY, N., CAI, Z., MASHOUF, S., REILLY, R. \& PIGNOL, J. P. (2011) Implications on clinical scenario of gold nanoparticle radiosensitization in regards to photon energy, nanoparticle size, concentration and location. Physics in Medicine and Biology, 56, 4631-4647.

LECHTMAN, E., MASHOUF, S., CHATTOPADHYAY, N., KELLER, B. M., LAI, P., CAI, Z., REILLY, R. M. \& PIGNOL, J. P. (2013) A Monte Carlo-based model of gold nanoparticle radiosensitization accounting for increased radiobiological effectiveness. Physics in Medicine and Biology, 58, 3075-3087.

LEUNG, M. K. K., CHOW, J. C. L., CHITHRANI, B. D., LEE, M. J. G., OMS, B. \& JAFFRAY, D. A. (2011) Irradiation of gold nanoparticles by $\mathrm{x}$-rays: Monte Carlo simulation of dose enhancements and the spatial properties of the secondary electrons production. Medical Physics, 38, 624-631. 
MAGGIORELLA, L., BAROUCH, G., DEVAUX, C., POTTIER, A., DEUTSCH, E., BOURHIS, J., BORGHI, E. \& LEVY, L. (2012) Nanoscale therapy with hafnium oxide nanoparticles Future Oncology, 8, 1167-1181.

MCMAHON, S. J., HYLAND, W. B., MUIR, M. F., COULTER, J. A., JAIN, S., BUTTERWORTH, K. T., SCHETTINO, G., DICKSON, G. R., HOUNSELL, A. R., O'SULLIVAN, J. M., PRISE, K. M., HIRST, D. G. \& CURRELL, F. J. (2011a) Biological consequences of nanoscale energy deposition near irradiated heavy atom nanoparticles. Scientific Reports, 1.

MCMAHON, S. J., HYLAND, W. B., MUIR, M. F., COULTER, J. A., JAIN, S., BUTTERWORTH, K. T., SCHETTINO, G., DICKSON, G. R., HOUNSELL, A. R., O'SULLIVAN, J. M., PRISE, K. M., HIRST, D. G. \& CURRELL, F. J. (2011b) Nanodosimetric effects of gold nanoparticles in megavoltage radiation therapy. Radiotherapy and Oncology, 100, 412-416.

MERCHANT, M. J., JEYNES, J. C. G., GRIME, G. W., PALITSIN, V., TULLIS, I. D. W., BARBER, P. R., VOJNOVIC, B., WEBB, R. P. \& KIRKBY, K. J. (2012) A Focused Scanning Vertical Beam for Charged Particle Irradiation of Living Cells with Single Counted Particles. Radiation Research, 178, 182-190. MISAWA, M. \& TAKAHASHI, J. (2011) Generation of reactive oxygen species induced by gold nanoparticles under X-rays and UV irradiations. Nanomedicine-Nanotechnology Biology and Medicine, 7, 604-614.

PATEL, P. C., GILJOHANN, D. A., SEFEROS, D. S. \& MIRKIN, C. A. (2008) Peptide antisense nanoparticles. Proceedings of the National Academy of Sciences of the United States of America, 105, 1722217226.

PECKYS, D. B. \& DE JONGE, N. (2011) Visualizing Gold Nanoparticle Uptake in Live Cells with Liquid Scanning Transmission Electron Microscopy. Nano Letters, 11, 1733-1738.

POLF, J. C., BRONK, L. F., DRIESSEN, W. H. P., ARAP, W., PASQUALINI, R. \& GILLIN, M. (2011) Enhanced relative biological effectiveness of proton radiotherapy in tumor cells with internalized gold nanoparticles. Applied Physics Letters, 98.

SEPAN, V. \& DAVIDKOVA, M. (2008) Impact of oxygen concentrations on yields of DNA damages caused by ionizing radiations. Journal of Physics: Conference Series, 101, 012015.

SHINOHARA, K., NAKANO, H. \& OHARA, H. (1996) Detection of Auger enhancement induced in HeLa cells labeled with iododeoxyuridine and irradiated with $150 \mathrm{kV}$ x-rays - Effects of cysteamine and dimethylsulfoxide. Acta Oncologica, 35, 869-875.

SICARD-ROSELLI, C., BRUN, E., MANON, G., BALDACCHINO, G., KELSEY, C., MCQUAID, H., POLIN, C., WARDLOW, N. \& CURRELL, F. (2014) A New Mechanism for Hydroxyl Radical Production in Irradiated Nanoparticle Solutions. Small.

SILVA, J. P. \& COUTINHO, O. P. (2010) Free Radicals in the regulation of damage and cell death - basic mechanisms and prevention Drug discoveries and therapeutics, 4, 144-167.

TOWNLEY, H. E. (2013) Applications of the Rare Earth Elements in Cancer Imaging and Therapy. Current Nanoscience, 9, 686-691.

TOWNLEY, H. E., KIM, J. \& DOBSON, P. J. (2012a) In vivo demonstration of enhanced radiotherapy using rare earth doped titania nanoparticles. Nanoscale, 4, 5043-5050.

TOWNLEY, H. E., RAPA, E., WAKEFIELD, G. \& DOBSON, P. J. (2012b) Nanoparticle augmented radiation treatment decreases cancer cell proliferation. Nanomedicine-Nanotechnology Biology and Medicine, 8, 526-536.

TSAI, D.-H., DELRIO, F. W., KEENE, A. M., TYNER, K. M., MACCUSPIE, R. I., CHO, T. J., ZACHARIAH, M. R. \& HACKLEY, V. A. (2011) Adsorption and Conformation of Serum Albumin Protein on Gold Nanoparticles Investigated Using Dimensional Measurements and in Situ Spectroscopic Methods. Langmuir, 27, 2464-2477.

TSAI, S.-W., CHEN, Y.-Y. \& LIAW, J.-W. (2008) Compound cellular imaging of laser scanning confocal microscopy by using gold nanoparticles and dyes Sensors, 8, 2306-2316.

WAZLEIN, C., SCIFONI, E., M, K. \& DURANTE, M. (2014) Simulations of dose enhancement for heavy atom nanoparticles irradiated by protons. Physics and Medicine and Biology, 59, 1441-1458.

ZIEGLER, J. F., ZIEGLER, M. D. \& BIERSACK, J. P. (2010) SRIM - The stopping and range of ions in matter. Nucl. Instrum. Methods B, 268, 1818-18 
